\title{
INVESTIGACIONES
}

\section{Los imaginarios sociales, el pensamiento crítico y la noviolencia, una forma para enfrentar el acoso escolar}

Social imaginaries, critical thinking, and nonviolence, a way to deal with school

\section{Clara Amaya Monje}

Universidad Nacional de Educación a Distancia (UNED). Liceo Quindío, Colombia

Telf.: (57) 3158480994. Correo electrónico: claraluama@yahoo.com.ar

\section{RESUMEN}

El objetivo principal es desarrollar en el estudiantado competencias ciudadanas que los lleven a respetar la diversidad de la comunidad educativa, reconociendo a sus miembros por sus condiciones y situaciones, y permitiendo la inclusión y erradicación del acoso escolar, en una institución pública del Quindío, Colombia. Se realizaron encuestas, grupos de discusión, entrevistas y talleres. Participaron estudiantes entre 11 y 14 años, padres, madres y profesorado. Los resultados muestran cómo los observadores del acoso escolar, interviniendo de manera noviolenta, pueden transformar los imaginarios sociales que legitiman la intimidación.

Palabras clave: acoso escolar, imaginarios sociales, pensamiento crítico, noviolencia, competencias ciudadanas.

\section{ABSTRACT}

The main goal is to develop civic competence among students to lead them to respect diversity in the educational community, acknowledging its members by their status and circumstances, allowing the acceptance and eradication of school bullying, inside a public institution of Quindío, Colombia. Polls, discussion groups, interviews and workshops were held. Students -between 11 and 14 -, their parents and teachers participated. The results show how spectators of bullying can transform the social imaginaries that enable the intimidation in a nonviolent way.

Key words: school's bullying, social imaginaries, critical thinking, nonviolence, citizenship skills. 


\section{INTRODUCCIÓN}

La violencia ha existido en la historia de la humanidad; violencia para sobrevivir, violencia para controlar el poder, violencia contra la dominación, violencia física, violencia verbal y psíquica, entre otras. La Organización Mundial de la Salud (OMS, 2002) clasifica la violencia según las siguientes categorías: "la violencia autoinfligida (comportamiento suicida y autolesiones), la violencia interpersonal (violencia familiar, que incluye menores, pareja y ancianos; así como violencia entre personas sin parentesco), la violencia colectiva (social, política y económica)" (p. 5). Los dos últimos tipos de violencia dificultan que en los grupos sociales se construyan ambientes democráticos (Forero-Londoño, 2011).

Dentro del tipo de violencia interpersonal se ubica la violencia escolar. Esta se compone de tres clases de manifestaciones que son clasificadas por Mockus y Cancino (2012) como: "Las agresiones escolares [...], los conflictos entre estudiantes [...] y la intimidación" (p. 17). Esta última, también denominada acoso u hostigamiento escolar, es el tema central de esta investigación, y se presenta en la interacción personal en cualquier contexto en el cual hacen presencia varias personas, de forma obligatoria y permanente (Valadez et al., 2011.), y se viven situaciones de maltrato (físico, verbal o relacional), repetida y sistemáticamente, hacia estudiantes que son considerados por el agresor/es más débiles o menos poderosos (Olweus, 1993; Roman y Murillo, 2011; Valadez, 2008).

No solamente en las Instituciones Educativas (IE) se vive el maltrato, sino en general en las sociedades, porque las personas que allí habitan se convierten en depósitos de “[...] un saber heredado, de lo ya instituido socialmente, a través de la asimetría existente en la sociedad en materia de imaginarios sociales, una asimetría que lleva a consolidar posiciones dominantes y también, al mismo tiempo, posiciones dominadas" (Baeza, 2011, p. 86). Estos imaginarios se expresan en lo simbólico de los lenguajes, generalmente de manera inconsciente. Los imaginarios son importantes para la construcción de convivencia social, aunque algunos de ellos orientan la generación de malestar, por lo que es necesario trabajar para transformar aquellos que no orientan al bienestar, para lo cual el pensamiento crítico y la noviolencia desempeñan un papel fundamental.

\subsection{INVESTIGACIONES ACERCA DEL TEMA}

Con relación al acoso escolar, existen estudios realizados en diferentes países, entre ellos España y 16 latinoamericanos (Argentina, Brasil, Colombia, Costa Rica, Cuba, Chile, Ecuador, El Salvador, Guatemala, México, Nicaragua, Panamá, Paraguay, Perú, República Dominicana y Uruguay). Dentro de las investigaciones hechas en España, existe un estudio en el cual determinaron formas de ejercer violencia profesorado-alumnado-profesorado. Participaron 4943 estudiantes con edades entre 12 y 17 años. Según los resultados obtenidos, Domínguez et al. (2013) encontraron que las conductas más utilizadas por el profesorado son "[...] bajar las notas y preferencia por determinado alumnado (30.3\%), manías y castigos injustificados (26.3\%) e ignorar a ciertos alumnos (21.7\%)" (p. 82). En lo referente a la violencia escolar del estudiantado hacia el profesorado, las acciones más comunes, según Domínguez et al. (2013) son "[...] hablar y comportarse de forma inadecuada dificultando las explicaciones del profesorado (42.8\%), falta de respeto y malos modales (14.6\%) e insultos (10.3\%)" (p. 82).

En Latinoamérica se encuentra un estudio cualitativo desarrollado en los 16 países mencionados en el párrafo anterior; su objetivo principal fue establecer la relación entre 
violencia escolar, maltrato escolar o bullying y desempeño académico en lectura y matemáticas. La muestra la conformaron 91223 estudiantes de $6^{\circ}$ grado (Román y Murillo, 2011). Dentro de los resultados este estudio pudo demostrar que:

La agresión más frecuente es el robo $(39,4 \%)$, seguida de la violencia verbal $(26,6 \%)$ y, por último, la violencia física $(16,5 \%)$. Aunque esta ordenación se mantiene en todos los países, las cifras son muy diferentes de uno a otro. [...] en Colombia más de la mitad de los alumnos de 6 grado dicen haber sufrido algún tipo de robo en el último mes, en Cuba solo lo afirma 1 de cada 10. (p. 44).

Teniendo en cuenta el objetivo principal del estudio en cuanto a la afectación que tiene el maltrato escolar en el rendimiento académico, se demuestra que en los estudiantes que hayan sido víctimas u observadores de tales situaciones se afecta de forma negativa su desempeño escolar en lectura y matemáticas. También se pudo comprobar que cuando en un establecimiento educativo el bullying es generalizado, este tiene poca o ninguna incidencia en el desempeño escolar (Román y Murillo, 2011).

Pasando al caso de Colombia, se encuentra una investigación realizada en Antioquia sobre naturalización de la intimidación. El objetivo de este estudio consistió en realizar la reflexión sobre los resultados de la investigación cualitativa: "Bullying: construcción de lo social en instituciones educativas, respuestas pedagógicas —estudio de casos_-". Dicho estudio fue llevado a cabo en tres Instituciones Educativas públicas y privadas de estratos bajos, medios y altos, de los municipios de Medellín y la Estrella, con estudiantes en edades entre los 11 y 15 años. En el proceso participaron docentes, directivos docentes, padres y madres de familia. El estudio dio cuenta de cómo el acoso, la agresión y la intimidación en las IE son vividas como algo natural y, por tanto, invisibilizadas (Ghiso y Ospina, 2010).

También en Colombia, en procesos de intervención, se encuentra el programa multicomponente Aulas en paz, que según Chaux (2007) persigue el siguiente propósito:

[...] prevenir la agresión y promover la convivencia pacífica con: 1) un currículo para el desarrollo de competencias ciudadanas en aula; 2) refuerzo extracurricular en grupos de dos niños/ as inicialmente agresivos y cuatro prosociales; 3 ) talleres, visitas y llamadas telefónicas para padres/ madres de familia. (p. 36).

Para la presente investigación se ha tenido en cuenta como fuente importante los resultados de las pruebas $\mathrm{SABER}^{1}$ que realiza el estado colombiano. El último informe ejecutivo de las pruebas SABER del grado $9^{\circ}$, emitido en el año 2013, afirma que el hostigamiento escolar en los colegios oficiales urbanos de Colombia fue vivido de la siguiente manera: víctima 31\%, intimidador 22\% y observador $66 \%$ (Instituto Colombiano para el Fomento de la Educación Superior, 2013).

Los anteriores datos oficiales son clara evidencia de que en Colombia los niños, niñas y jóvenes actuales presentan bajos niveles de adquisición de habilidades que se requieren para el desarrollo de las competencias ciudadanas, por lo cual es relevante que se actúe de

El propósito principal de las denominadas pruebas SABER $3^{\circ}, 5^{\circ}$ y $9^{\circ}$ es contribuir al mejoramiento de la calidad de la educación colombiana, mediante la realización de medidas periódicas del desarrollo de competencias de los estudiantes de educación básica, como indicador de calidad del sistema educativo. http://www.mineducacion.gov.co/1621/w3article-244735.html 
manera pronta y eficaz con el propósito de trabajar ante esta delicada problemática para evitar que se sigan generando altos costos sociales y económicos, pues, como lo afirman Feldman, Donato y Wright (2013) “ [...] los jóvenes que fueron intimidados eran más propensos a desarrollar ansiedad generalizada y el trastorno de pánico como adultos; mientras que al intimidar a las víctimas tenían más probabilidades de sufrir posteriormente depresión y tendencias suicidas" (p. 51). Es de advertir que si se quiere que las generaciones más jóvenes no sigan repitiendo la violencia que, de muchas formas, les ha tocado vivir, deben ser vistos y tenidos en cuenta no como parte del problema, sino como parte de la solución.

Cabe anotar que esta investigación cobra especial relevancia porque muestra cómo el desarrollo del pensamiento crítico, que es parte fundamental de las competencias ciudadanas, es un elemento esencial para develar los imaginarios sociales que hacen presencia en la intimidación escolar. Por lo cual es trascendente crear alternativas noviolentas que posibiliten la transformación de algunos imaginarios sociales, para lograr erradicar el acoso escolar y, por consiguiente, desarrollar la construcción de competencias ciudadanas.

\subsection{REFERENTES CONCEPTUALES}

Dentro del acoso escolar ya definido en la introducción, es importante tener en cuenta otros conceptos que se hacen parte de este, entre ellos: acosador, víctima, observador o espectador. Loredo, Perea y López (2008) definen que: “el acosador (“bully”), es el que ejerce la violencia generalmente sobre un igual. La víctima es un individuo de la misma comunidad, quien por diversas condiciones suele mostrar inferioridad física, psicológica o social" (p. 212). Estas personas presentan baja autoestima, sentimientos de inferioridad y elevado resentimiento, y consideran además como algo natural el maltrato efectuado por algunos de sus iguales (Cowie y Fernández, 2006). En cuanto al observador o testigo, García (2009) determina a las personas que ejercen este rol como: "los y las compañeras que presencian los actos de violencia escolar [...]" (p. 4), algunos se muestran indiferentes, otros apoyan de alguna manera a la víctima.

Igualmente, se incluyen otros conceptos que son fundamentales para el propósito de la investigación, tales como: pensamiento crítico, imaginarios sociales, noviolencia, diversidad y competencias ciudadanas. Respecto a pensamiento crítico, Villarini (s.f.) lo define como "la capacidad del pensamiento para examinarse y evaluarse a sí mismo (el pensamiento propio o el de los otros). [...] La capacidad para el pensamiento crítico surge de la metacognición" (p. 39). Además, el pensamiento crítico "como parte las competencias cognitivas, debe estar presente para formar ciudadanos con capacidad de cuestionar la información, las creencias, las ideologías y las relaciones de poder que hacen parte de las dinámicas de una sociedad" (Ruiz y Chaux, s.f., p. 23).

Con relación a los imaginarios sociales, Pintos $(2004,2005,2014)$ aclara que estos no son representaciones, sino esquemas construidos socialmente, los cuales permiten a los miembros de una sociedad percibir, explicar e intervenir en lo que ellos tengan como realidad. Además, habla de sociedades policontexturales y pluriversas, puesto que considera que no son realidades ni contextos únicos y estáticos, sino que estos son cambiantes y diversos.

En lo concerniente a diversidad, Gento (2006) expresa lo siguiente: 
La diversidad, en sentido antropológico, hace referencia a la peculiaridad de cada persona, en cualquiera de las dimensiones y rasgos que la configuran, así como a cuantas circunstancias rodean su propia existencia. Caben, por tanto, en ella diferenciaciones relativas a: rasgos individuales: físicos, psíquicos o comportamentales. Rasgos de carácter social: cultura, condiciones sociales, raza, religión, etc. (p. 15).

Dentro del concepto de noviolencia, Fernández (2003) señala que se trata de

“[...] formas que rechazan la pasividad — dirigida a aguantar — y reivindica un camino creativo para construir mejores realidades. Este camino nos plantea resistir a las formas de violencia, desaprender la violencia, e inventar nuevas formas de cambio para transformar la realidad". (p. 42).

Por último, el concepto de competencias ciudadanas, que se debe trabajar desde la perspectiva de los derechos y es definido por Colombia aprende (2011) como: "una serie de conocimientos, actitudes y habilidades comunicativas, emocionales, cognitivas e integradoras que funcionan de manera articulada para que todas las personas seamos sujetos sociales activos de derechos" (p. 22).

\subsection{HIPÓTESIS DE LA INVESTIGACIÓN}

La comunidad educativa del departamento del Quindío está presentando bajos niveles de desarrollo de habilidades para la construcción de competencias ciudadanas.

\subsection{PREGUNTA DE INVESTIGACIÓN}

El acoso escolar como una forma de intolerancia a la diversidad ¿de qué manera se está manifestando dentro de las Instituciones Educativas (IE) del departamento ${ }^{2}$ del Quindío y qué están haciendo las IE para afrontar la problemática?

\subsection{OBJETIVO GENERAL}

Desarrollar en el estudiantado competencias ciudadanas que los lleven a respetar la diversidad de la comunidad educativa, reconociendo a sus miembros por sus condiciones y situaciones y permitiendo la inclusión y erradicación del acoso escolar.

\subsection{OBJETIVOS ESPECÍFICOS}

1) Explorar situaciones de acoso escolar y posibles roles entre estudiante-estudiante, profesor/a-estudiante, que se han venido presentando durante el año lectivo. 2) Identificar acciones llevadas a cabo por las IE para afrontar la problemática del acoso. 3) Brindar a algunos miembros de la comunidad educativa herramientas teórico prácticas sobre pensamiento crítico, imaginarios sociales y noviolencia que permitan construir e implementar acciones noviolentas ante el acoso escolar.

Se excluye el municipio de Armenia por encontrarse certificado, por lo cual es el único que no depende administrativamente de la gobernación del Quindío. 


\section{METODOLOGÍA}

Esta investigación fue realizada dentro del enfoque mixto, que es definido por Hernández, Fernández y Baptista (2010) como “[...] un conjunto de procesos sistemáticos, empíricos y críticos de investigación e implican la recolección y el análisis de datos cuantitativos y cualitativos, así como su integración y discusión conjunta, para realizar inferencias [...]" (p. 546). Además, los componentes fueron desarrollados de manera concurrente, es decir "se aplican ambos métodos de manera simultánea (los datos cuantitativos y cualitativos se recolectan y analizan más o menos al mismo tiempo) [...]” (Hernández et al., 2010, p. 559). Una vez recogidos los datos cuantitativos y cualitativos, se analizaron y discutieron de manera independiente, y con los resultados arrojados se hizo triangulación para llegar a metainferencias. En cuanto al diseño de la investigación, este fue no experimental.

Igualmente, se trabajó desde el marco de la investigación aplicada, que Vargas (2009), desde la epistemología, considera que:

[...] esta expresión está en la base de distinciones tales como 'saber y hacer', 'conocimiento y práctica', 'explicación y aplicación', 'verdad y acción'. '[y es] un enlace importante entre ciencia y sociedad. Con ella, los conocimientos son devueltos a las áreas de demanda, ubicadas en el contexto, donde se da la situación que debe ser intervenida, mejorada o transformada'. (pp. 160-163).

Son parte de esta investigación estudiantes cuyas edades fluctúan entre 11 y 14 años, quienes se encontraban matriculados en $\operatorname{los}$ grados 7 u 8 de secundaria; docentes y directivos docentes; y padres, madres o acudientes del estudiantado que conformaron este estudio. Los participantes formaban parte de la comunidad educativa de diferentes IE del departamento del Quindío, Colombia, a los cuales se pudo acceder mediante cartas dirigidas a los rectores y consentimientos informados que explicaban el objetivo investigativo del estudio, al igual que la confidencialidad y el anonimato.

Para la recolección de la información se utilizaron diferentes procedimientos, entre ellos de interrogación escrita, adaptando el cuestionario de secundaria de 12 a 16 años que aparece en el informe del defensor del pueblo, la Organización de Estados Iberoamericanos para la Educación, la Ciencia y la Cultura (OIE, 2007). En cuanto al procedimiento de interrogación oral, las técnicas utilizadas fueron entrevistas y grupos de discusión.

Es así que el cuestionario constó de 20 ítems. Los 17 primeros dirigidos a indagar sobre la primera parte de la pregunta de investigación, relacionada a cómo se está manifestando el hostigamiento en la IE, y las tres restantes a preguntar qué están haciendo las IE para enfrentar la problemática. Todos los ítems tuvieron una evaluación mediante escala tipo Likert, puntuando de 1 (nunca), 2 (rara vez), 3 (a veces), 4 (muchas veces) y 5 (siempre). Fueron tenidas en cuenta como situaciones de intimidación las puntuaciones 4 y 5 , asignadas por cada estamento.

Con el propósito de evaluar la fiabilidad de los ítems se manejó el coeficiente de Alfa de Cronbach, que arrojó los siguientes resultados: para el pre test fue de 0,812, el del postest de 0,803 y para la información completa fue de 0,809. Lo que quiere decir que el nivel de correlación de las variables en el instrumento es adecuado con el instrumento como un todo. Esto denota a priori una buena consistencia interna del instrumento (García, González y Ballesteros, 2001; Hernández, Fernández y Baptista, 2010). 
Durante la investigación se efectuaron dos mediciones, una al iniciar proceso y otra al finalizar. En cada medición se estudió una muestra de la misma población, a quienes se les explicó que solo se debían tener en cuenta los últimos tres meses de acuerdo con la fecha de aplicación del instrumento. En la primera medición participaron en las encuestas 185 estudiantes, 50 docentes y directivos docentes y 87 padres, madres o acudientes. Debido a que en el pretest el nivel de homogeneidad fue alto dentro de cada uno de los estamentos encuestados (estudiantes, padres, profesorado), y según García et al. (2001) el tamaño de la muestra "depende del grado de homogeneidad de la población, cuanto más homogénea sea, más sencillo resulta alcanzar muestras representativas y con un menor tamaño" (p. 210), se justificó que para el postest se redujera el tamaño de la muestra sin que esto afectara estadísticamente su representatividad. Fue así como en la segunda medición fueron encuestados 31 estudiantes, 11 docentes y directivos docentes y 21 padres, madres o acudientes, quienes habían participado en el pretest. Para el análisis de las encuestas se utilizó el programa estadístico SPSS. Dicho análisis se realizó utilizando tablas de contingencias y el coeficiente Chi-Cuadrado de Pearson para cada momento en que se aplicó la encuesta (pretest y postest).

En cuanto a la información cualitativa, esta también se recogió dos veces. En la primera ocasión se realizaron tres grupos de discusión heterogéneos, compuestos cada uno por tres estudiantes, dos docentes y dos padres, madres o acudientes. Fueron entrevistados 10 estudiantes, 10 docentes y directivos docentes y 10 padres, madres o acudientes. En la segunda oportunidad se constituyeron cuatro grupos de discusión heterogéneos, compuestos cada uno por cinco estudiantes, dos docentes y dos padres, madres o acudientes. Para el análisis de esta información se utilizó el programa Atlas ti.

Para llevar a cabo la intervención se realizaron talleres que incluían actividades lúdicas, conversatorios y video-foros. Participaron cinco estudiantes de cada uno de los tres grupos a intervenir, que fueron seleccionados por otros estudiantes y docentes. A estos estudiantes se les brindó herramientas teóricas y prácticas sobre acoso escolar, pensamiento crítico, imaginarios sociales y noviolencia. Dichos estudiantes eran identificados como observadores en la dinámica del hostigamiento escolar y tenían, además, que cumplir con otras condiciones, puesto que ellos desempeñan un papel fundamental debido al poder que tienen en las situaciones de acoso. Tal como Chaux (2012) lo explica, "ser consciente de este poder que tienen los observadores genera un sentimiento importante de responsabilidad social y la comprensión de que no hacer nada es, en última, ser cómplice de lo que está sucediendo" (p. 142). Dentro de las otras condiciones definidas se encuentran tener habilidades tales como: buenos niveles de empatía, compromiso con el proceso a realizar; y capacidad para hablar y actuar frente a su grupo en lo relacionado a la intimidación escolar, pues debían liderar en el aula la intervención ante situaciones de intimidación, como también proponer actividades a ser desarrolladas con todo su grupo. Para el desarrollo de dichas actividades estuvieron apoyados por docentes escogidos por ellos mismos.

\section{RESULTADOS}

En la Tabla 1 se muestra un resumen en el que se fundamenta la asociación entre las variables. El análisis se realizó utilizando tablas de contingencias y el coeficiente ChiCuadrado de Pearson para cada momento en que se aplicó la encuesta: pretest y postest. 
Estudios Pedagógicos XLII, N 3: 27-48, 2016

LOS IMAGINARIOS SOCIALES, EL PENSAMIENTO CRÍTICO Y LA NOVIOLENCIA, UNA FORMA PARA ENFRENTAR EL ACOSO ESCOLAR

En el pretest, la mayoría (95\%) de los constructos presentan asociación estadísticamente significativa con los estamentos de donde provienen las respuestas, es decir, existe un alto grado de acuerdo al interior de los estamentos (padres, estudiantes, profesorado) respecto a la connotación que le daban a situaciones vividas por el estudiantado, las cuales no eran reconocidas como acoso escolar por los miembros de cada estamento, pero que sí fueron reconocidas como tales en las entrevistas y grupos de discusión (Tabla 5).

Tabla1. Variables con los Chi-Cuadrados de Pearson del análisis de asociación entre estamentos

\begin{tabular}{|l|c|c|c|c|}
\hline Variable & \multicolumn{2}{|c|}{ Pretest } & \multicolumn{2}{c|}{ Postest } \\
\hline & $\chi^{2}$ & Sig. & $\chi^{2}$ & Sig. \\
\hline No dejan lo/la participar & 54.824 & 0,000 & 13.766 & 0,088 \\
\hline Hablan mal de ella/él & 37.401 & 0,000 & 8.620 & 0,196 \\
\hline Lo/la rechazan & 57.756 & 0,000 & 19.881 & 0,011 \\
\hline Lo/la insultan & 28.959 & 0,001 & 12.646 & 0,049 \\
\hline Le dicen apodos ridículos u ofensivos & 82.460 & 0,000 & 19.913 & 0,011 \\
\hline Se burlan & 67.245 & 0,000 & 9.477 & 0,148 \\
\hline Le roban cosas & 68.456 & 0,000 & 14.527 & 0,150 \\
\hline Le dañan cosas & 89.970 & 0,000 & 9.471 & 0,149 \\
\hline Le esconden cosas & 38.999 & 0,000 & 7.946 & 0,439 \\
\hline Lo/la amenazan para meterle miedo & 47.855 & 0,000 & 9.238 & 0,161 \\
\hline Le pegan & 36.852 & 0,000 & 15.242 & 0,018 \\
\hline La/lo amenazan para obligarlo a hacer cosas que no quiere & 126.705 & 0,000 & 9.237 & 0,055 \\
\hline Profesores hacen alguna de las cosas anteriores & 28.975 & 0,000 & 6.762 & 0,343 \\
\hline Uso de medios electrónicos para hacer daño & 14.923 & 0,061 & 11.025 & 0,026 \\
\hline Informar a un familiar sobre acoso escolar & 54.058 & 0,000 & 16.111 & 0,096 \\
\hline Ver cometer acoso escolar & 39.331 & 0,000 & 20.284 & 0,009 \\
\hline Comete acoso escolar & 37.871 & 0,000 & 30.145 & 0,001 \\
\hline Información del colegio sobre ley de convivencia & 53.409 & 0,000 & 25.504 & 0,004 \\
\hline El colegio realiza actividades para mejorar convivencia & 43.966 & 0,000 & 19.577 & 0,034 \\
\hline $\begin{array}{l}\text { El colegio informa sobre funcionamiento de comité de } \\
\text { convivencia }\end{array}$ & 31.284 & 0,000 & 16.599 & 0,084 \\
\hline
\end{tabular}

Las tablas 2 a 8 responden a la primera parte de la pregunta de investigación: cómo se está manifestando el acoso escolar en las IE. Para ello se indagó acerca de diferentes situaciones mediante las cuales estudiantes son intimidados. Para el análisis se tuvieron 
en cuenta los tres ítems que obtuvieron mayores frecuencias por parte del estudiantado, ya que este estamento es el directamente afectado. No obstante, las respuestas de padres y profesorado fueron tenidas en cuenta para la posible confirmación o contradicción de las situaciones manifestadas: el robo es la primera situación con mayor frecuencia; la segunda, decirles apodos ridículos; y la tercera, hablar mal de ellos. Teniendo en cuenta que por grupo el número de estudiantes de secundaria oscila entre 32 y 40 estudiantes (Ministerio de Educación Nacional [MEN], 2002), se puede decir que en promedio 5 estudiantes por aula son víctimas de robo, 5 son víctimas de apodos (tablas 2 y 3 ) y 4 estudiantes son acosados hablando mal de ellos (Tabla 4).

En cuanto al robo (Tabla 2), las cifras fueron las similares en el pretest (14\%) y postest (16\%) del estudiantado, y fueron confirmadas por las respuestas de los padres y principalmente del profesorado con un alto porcentaje en el pretest (28\%) y en el postest (45\%). No obstante, esta situación no fue mencionada en las entrevistas y grupos de discusión (Tabla 5).

Tabla 2. Acosado por medio de robo

\begin{tabular}{|l|c|c|c|c|}
\hline \multirow{2}{*}{\multicolumn{1}{|c|}{ Estamento }} & \multicolumn{2}{c|}{ Pretest } & \multicolumn{2}{c|}{ Postest } \\
\cline { 2 - 5 } & Frecuencia & Porcentaje & Frecuencia & Porcentaje \\
\hline Padres, madres o acudientes & 21 & 24 & 3 & 14 \\
\hline Estudiantado & 25 & 14 & 5 & 16 \\
\hline Profesorado & 14 & 28 & 5 & 45 \\
\hline
\end{tabular}

Respecto a los apodos (Tabla 3), las cifras del pretest (13\%) y postest (16\%) del estudiantado son similares a las de los padres. Además son confirmadas por el profesorado con altas cifras: pretest (28\%) y postest (45\%). Estas situaciones también fueron evidentes en las entrevistas y grupos de discusión (Tabla 5) previas a la intervención. Aunque los resultados del postest no son coincidentes con los resultados de los grupos de discusión, ni con las entrevistas posteriores a la intervención (Tabla 5), puesto que allí se afirmó que esta situación había mejorado.

Tabla 3. Acoso por medio de apodos

\begin{tabular}{|l|c|c|c|c|}
\hline \multirow{2}{*}{\multicolumn{1}{|c|}{ Estamento }} & \multicolumn{2}{c|}{ Pretest } & \multicolumn{2}{c|}{ Postest } \\
\cline { 2 - 5 } & Frecuencia & Porcentaje & Frecuencia & Porcentaje \\
\hline Padres, madres o acudientes & 13 & 15 & 2 & 10 \\
\hline Estudiantado & 24 & 13 & 5 & 16 \\
\hline Profesorado & 14 & 28 & 5 & 45 \\
\hline
\end{tabular}


Con relación a que hablan mal de ellas y ellos (Tabla 4), las cifras del pretest (11\%) del estudiantado son similares a las de los padres y el profesorado. Las cifras del postest (6\%) del estudiantado bajan prácticamente a la mitad y son similares a las de los padres, pero no coinciden con las del profesorado, ya que el resultado fue cero. Es decir, el profesorado ya no catalogó esta situación como hostigamiento y tampoco fue nombrada en los grupos de discusión y entrevistas (Tabla 5).

Tabla 4. Acoso por medio de hablar mal de ellos/as

\begin{tabular}{|l|c|c|c|c|}
\hline \multirow{2}{*}{ Estamento } & \multicolumn{2}{c|}{ Pretest } & \multicolumn{2}{c|}{ Postest } \\
\cline { 2 - 5 } & Frecuencia & Porcentaje & Frecuencia & Porcentaje \\
\hline Padres, madres o acudientes & 9 & 10 & 1 & 5 \\
\hline Estudiantado & 21 & 11 & 2 & 6 \\
\hline Profesorado & 6 & 12 & 0 & 0 \\
\hline
\end{tabular}

Dentro de lo expresado por las y los participantes en las entrevistas y grupos de discusión (Tabla 5), en el pretest se evidenció el acoso mediante apodos, aunque algunos consideraban que eso era un juego, mientras las víctimas expresaron no sentirse en paz. También fue reiterativo sobre el papel que está desempeñando actualmente la familia en cuanto al bajo compromiso con la formación en valores de los hijos. Es importante destacar el hostigamiento manifestado entre algunos/as estudiantes-profesores-estudiantes. Según los resultados pos-intervención, este proceso contribuyó a aspectos como que gran parte de los participantes comprendieran el concepto de acoso escolar y, que el respeto por el otro fuera una prioridad para evitar o erradicar la intimidación, lo cual no dependía solo de algunos miembros del grupo sino de todos. Sin embargo, el hostigamiento entre algunos/as profesores-estudiantes-profesores en los resultados continuó presente.

Tabla 5. Entrevistas y grupos de discusión sobre situaciones de intimidación escolar

\begin{tabular}{|l|l|}
\hline \multicolumn{1}{|c|}{ Pre-intervención } & \multicolumn{1}{|c|}{ Pos-intervención } \\
\hline $\begin{array}{l}\text { Es que los apodos que les decimos a los } \\
\text { compañeros es solo por molestar. }\end{array}$ & $\begin{array}{l}\text { Ya casi nadie molesta con los apodos. Poco a } \\
\text { poco vamos aprendiendo a respetar. }\end{array}$ \\
\hline $\begin{array}{l}\text { Hay peladitos muy montadores y no lo dejan a } \\
\text { uno en paz diciéndole apodos. }\end{array}$ & $\begin{array}{l}\text { Él tiene derecho a la autodeterminación de ser } \\
\text { gay o no. }\end{array}$ \\
\hline $\begin{array}{l}\text { Hay compañeros muy fregados y se la montan a } \\
\text { los profesores que les exigen las tareas. }\end{array}$ & $\begin{array}{l}\text { No soy del grupo de las capacitaciones del } \\
\text { bullying, pero las actividades en el salón me } \\
\text { aclararon mucho lo que significa y qué } \\
\text { debemos y qué no debemos hacer. }\end{array}$ \\
\hline
\end{tabular}


A esa profesora cuando llega le tiran papeles y
le dicen cosas. Pero es que ella no lo respeta a
uno, ni se hace respetar.

Creo que una función principal de las familias es que eduquemos con valores a nuestros hijos. Pero hoy en día en, muchas familias, los que mandan son los hijos.

En mí salón una compañera que cuando habla, los compañeros la empiezan a silbar, a gritarle.

Se han hecho reuniones con orientación, coordinación, estudiantado y profesorado con los cuales se ha presentado esa problemática, pero no se han visto cambios de ninguna de las partes.
Burlarse de la forma como se viste una persona es no respetarle el libre desarrollo de la personalidad que tanto exigimos.

Son procesos culturales demorados, pero sí ha habido cambios positivos. Se preocupan más porque sus compañeros respeten. Aunque la relación con algunos profesores sigue igual de maluca.

Los que estamos en estos talleres la idea es que nos unamos para evitar. Pero cuando lo hagamos no nos sulfuremos.

Por muchas actividades que se hagan, no sirven porque los profesores siempre tienen la razón.

En lo concerniente a estudiantes observadores, según el pretest, en promedio 7 de cada grupo reconocen ser testigos de situaciones de intimidación. En el pretest las respuestas de padres y profesorado confirman lo expresado por el estudiantado. Por su parte, en el postest baja el número según los estudiantes y el profesorado confirma las respuestas del estudiantado. No obstante, según los padres ninguno de sus hijos/as ha sido testigo de acoso en el periodo tenido en cuenta en el postest.

Tabla 6. Estudiantes que observan situaciones de intimidación

\begin{tabular}{|l|c|c|c|c|}
\hline \multirow{2}{*}{\multicolumn{2}{|c}{ Estamento }} & \multicolumn{2}{c|}{ Pretest } & \multicolumn{2}{c|}{ Postest } \\
\cline { 2 - 5 } & Frecuencia & Porcentaje & Frecuencia & Porcentaje \\
\hline Padres, madres o acudientes & 18 & 21 & 0 & 0 \\
\hline Estudiantado & 36 & 19 & 4 & 13 \\
\hline Profesorado & 8 & 16 & 2 & 18 \\
\hline
\end{tabular}

Con relación a quienes realizan hostigamiento, según el pretest, en promedio 4 estudiantes de cada grupo se reconocen como tales, siendo estas respuestas confirmadas por los padres y el profesorado. Según el postest, en promedio 1 estudiante por grupo se reconoce como acosador, siendo esto confirmado por el profesorado, aunque ninguno de los padres identificó a su hija hijo como hostigador en el periodo tenido en cuenta en el postest. 
Tabla 7. Estudiantes identificados porque hacen acoso escolar

\begin{tabular}{|l|c|c|c|c|}
\hline \multirow{2}{*}{ Estamento } & \multicolumn{2}{|c|}{ Pretest } & \multicolumn{2}{c|}{ Postest } \\
\cline { 2 - 5 } & Frecuencia & Porcentaje & Frecuencia & Porcentaje \\
\hline Padres, madres o acudientes & 6 & 7 & 0 & 0 \\
\hline Estudiantado & 19 & 10 & 1 & 3 \\
\hline Profesorado & 10 & 20 & 2 & 18 \\
\hline
\end{tabular}

Según los resultados del pretest, en promedio 3 estudiantes por grupo expresaron que algún profesor realiza hostigamiento hacia alguien del estudiantado. Pero tuvo baja coincidencia con las respuestas de los padres y profesorado, ya que el número de frecuencias estuvo muy por debajo de las del estudiantado. Aunque las frecuencias del estudiantado aparentemente no fueron tan altas, es importante destacar la situación, más aún porque en las encuestas y entrevistas fue también expresada (Tabla 5). En cuanto al postest, en promedio 2 estudiantes por grupo identificaron a algún/a profesora como hostigador/a, siendo esto corroborado por los padres, pero contrario a las respuestas del profesorado, dado que en este estamento la frecuencia fue 0 .

Tabla 8. Profesores/as identificados porque hacen acoso escolar

\begin{tabular}{|l|c|c|c|c|}
\hline \multirow{2}{*}{ Estamento } & \multicolumn{2}{|c|}{ Pretest } & \multicolumn{2}{c|}{ Postest } \\
\cline { 2 - 5 } & Frecuencia & Porcentaje & Frecuencia & Porcentaje \\
\hline Padres, madres o acudientes & 3 & 3 & 1 & 5 \\
\hline Estudiantado & 13 & 7 & 2 & 6 \\
\hline Profesorado & 2 & 4 & 0 & 0 \\
\hline
\end{tabular}

Las tablas 9, 10, 11 y 12 responden a la segunda parte de la pregunta de investigación: qué hacen las IE para afrontar esta problemática. Según los resultados del pretest y postest, se puede decir que entre el $45 \%$ y el $100 \%$ de los participantes afirmaron que las IE sí realizan actividades para mejorar la convivencia escolar. También que les han informado sobre el funcionamiento del comité de convivencia escolar y sobre la ley del sistema nacional de convivencia. Dentro de las acciones más realizadas por parte de las IE (Tabla 12), según lo expresado en la preintervención y posintervención, se encuentran: citar a los padres, realizar convivencias, talleres, charlas y capacitaciones. 
Tabla 9. El colegio realiza actividades para mejorar la convivencia escolar

\begin{tabular}{|l|c|c|c|c|}
\hline \multirow{2}{*}{ Estamento } & \multicolumn{2}{c|}{ Pretest } & \multicolumn{2}{c|}{ Postest } \\
\cline { 2 - 5 } & Frecuencia & Porcentaje & Frecuencia & Porcentaje \\
\hline Padres, madres o acudientes & 48 & 55 & 14 & 67 \\
\hline Estudiantado & 81 & 44 & 14 & 45 \\
\hline Profesorado & 24 & 48 & 5 & 45 \\
\hline
\end{tabular}

Tabla 10. El colegio nos informa acerca del funcionamiento del comité de convivencia escolar

\begin{tabular}{|l|c|c|c|c|}
\hline \multirow{2}{*}{\multicolumn{1}{|c|}{ Estamento }} & \multicolumn{2}{c|}{ Pretest } & \multicolumn{2}{c|}{ Postest } \\
\cline { 2 - 5 } & Frecuencia & Porcentaje & Frecuencia & Porcentaje \\
\hline Padres, madres o acudientes & 44 & 51 & 15 & 71 \\
\hline Estudiantado & 90 & 49 & 18 & 58 \\
\hline Profesorado & 35 & 70 & 11 & 100 \\
\hline
\end{tabular}

Tabla 11. En el colegio nos han informado sobre la ley del sistema nacional de convivencia escolar

\begin{tabular}{|l|c|c|c|c|}
\hline \multirow{2}{*}{\multicolumn{1}{|c|}{ Estamento }} & \multicolumn{2}{c|}{ Pretest } & \multicolumn{2}{c|}{ Postest } \\
\cline { 2 - 5 } & Frecuencia & Porcentaje & Frecuencia & Porcentaje \\
\hline Padres, madres o acudientes & 31 & 36 & 13 & 62 \\
\hline Estudiantado & 84 & 45 & 14 & 45 \\
\hline Profesorado & 41 & 82 & 10 & 91 \\
\hline
\end{tabular}

Tabla 12. Realización de acciones en las IE para evitar el acoso escolar

\section{Respuestas en entrevistas y grupos de discusión}

Citar al acudiente, pero algunos no vienen o mandan a la abuelita, tía o vecino, porque ellos dicen estar trabajando.

Han hecho convivencias y charlas, pero las hacen una vez al año y uno cambia los primeros días, y después sigue igual.

En las capacitaciones nos hablan de los derechos que los muchachos tienen. Pero si no se les forma en valores, nada hacemos. 
También se presentan otros aspectos que son necesarios para alcanzar los objetivos de la investigación. Entre ellos identificación de sentimientos y emociones (Tabla 13) que permiten hacer un acercamiento a las habilidades emocionales que son parte constitutiva de las competencias ciudadanas. Dentro de los sentimientos y emociones expresadas se encuentran: la rabia, el pesar, la depresión, el temor, la angustia, la indignación, la impotencia y la tristeza. Es importante destacar la idea suicida de un/a participante.

Tabla 13. Sentimientos y emociones generados por el acoso escolar

\begin{tabular}{|l|}
\hline \multicolumn{1}{|c|}{ Respuestas en Entrevistas y grupos de discusión } \\
\hline Me da rabia, a veces le contesto a esa profesora. \\
\hline Yo me quería suicidar. \\
\hline Me da pesar cuando a esa profesora le tiran papeles. \\
\hline Me siento mal, deprimido, no quiero volver a ese colegio. \\
\hline Temor de ser agredido en cualquier momento porque dicen que soy gay. \\
\hline Me da angustia e indignación ver que esa persona está sufriendo. \\
\hline Impotencia por no poder lograr cambios. \\
\hline Tristeza de que los padres no eduquen bien a sus hijos para que respeten. \\
\hline Me da temor mandar mi hijo al colegio. \\
\hline
\end{tabular}

En las tablas 14 y 15 se encuentran respuestas que sirven de aproximación a las posibles causas e imaginarios de los participantes para que se presente la intimidación: se piensa que el acoso escolar es un juego, que es algo natural, porque los estudiantes presentan capacidades diversas, porque no hay que dejarse de nadie, por la falta de valores, porque la víctima se lo merece, porque la violencia intrafamiliar lo genera, por estrés en algunos docentes por presión en las IE, por falta de herramientas metodológicas y de manejo de conflictos por parte de los docentes, porque las personas manifiestan la homosexualidad públicamente y porque no se respetan los acuerdos.

Tabla 14. Causas del acoso escolar

\begin{tabular}{|l|}
\hline \multicolumn{1}{|c|}{ Respuestas en Entrevistas y grupos de discusión } \\
\hline Porque ellos piensan que eso es un juego. \\
\hline Falta de valores, de ética. Unos se creen mejores que otros. \\
\hline En la casa no los tratan bien y se desquitan en el colegio. \\
\hline $\begin{array}{l}\text { El acoso ha existido y siempre existirá, eso hace parte de los seres humanos. Lo que } \\
\text { pasa es que ahora por los medios de comunicación la gente se entera más. }\end{array}$ \\
\hline $\begin{array}{l}\text { Si lo ejercen algunos docentes puede ser por el estrés, problemas de presión en la IE, } \\
\text { falta de herramientas metodológicas y de manejo de conflictos. }\end{array}$ \\
\hline Porque los estudiantes muestran necesidades educativas especiales. \\
\hline Uno no se debe dejar de nadie. Eso me lo enseñaron en la casa desde que era chiquito. \\
\hline
\end{tabular}


Tabla 15. Algunos imaginarios evidenciados en las capacitaciones

\begin{tabular}{|l|l|}
\hline \multicolumn{1}{|c|}{ Actividad } & \multicolumn{1}{c|}{ Lo expresado por los y las participantes } \\
\hline Video-foro & $\begin{array}{l}\text { Es que esos niños especiales como el del video, es mejor que estudien en } \\
\text { otro colegio para que no se burlen de ellos. } \\
\text { Esa vieja se lo tenía merecido por perra y pa más... fea. }\end{array}$ \\
\hline Juego de roles & $\begin{array}{l}\text { Si hubiera sabido que la pareja de esa vieja era otra mujer, no hubiera ido a } \\
\text { ese matrimonio. } \\
\text { Si de verdad yo hubiera sido el papá de esa vieja no hubiera dejado que se } \\
\text { volviera machorra. }\end{array}$ \\
\hline Socio-dramas & $\begin{array}{l}\text { No estoy de acuerdo que cuando el novio del profesor fue a dejarlo al colegio } \\
\text { se despidieran de beso delante de todos, porque ellos son los primeros que } \\
\text { tienen que respetar. } \\
\text { Es que si la pareja del profesor fuera una vieja sería normal que le diera un } \\
\text { beso al despedirse. }\end{array}$ \\
\hline Juegos cooperativos & $\begin{array}{l}\text { Aunque todos los líderes de los grupos acordamos cómo íbamos a votar, yo } \\
\text { me voltee para que mi equipo ganara más puntos. Reconozco que no respete } \\
\text { una de las reglas del juego y por eso a la final perdimos todos. }\end{array}$ \\
\hline
\end{tabular}

\section{DISCUSIÓN}

Tomando como base los resultados obtenidos, se confirma la hipótesis respecto a los bajos niveles de desarrollo de habilidades para la construcción de competencias ciudadanas que se están presentando en las IE del Quindío, puesto que se pudo comprobar la presencia de comportamientos negativos hacia la empatía, cuidado y respeto tanto por la otra y el otro como por sus pertenencias, al igual que actitudes contrarias a la aceptación de la diversidad.

Para presentar la discusión a la pregunta de investigación, esta se organizó en dos partes: la primera hace referencia a cómo se está manifestando el acoso escolar y la segunda a qué están haciendo las IE para afrontar esta problemática. El robo es la primera situación con mayor frecuencia; la segunda, decir apodos ridículos; y la tercera, hablar mal de alguien del estudiantado. En cuanto al robo, coincide con los resultados del estudio hecho en escuelas latinoamericanas (Roman y Murillo, 2011), en el cual Colombia también se encuentra en primer lugar en hechos de robo.

Las situaciones de robo y hablar mal de un compañero o compañera son catalogadas formas indirectas de hostigamiento, Collell y Escudé (2006) explican cómo afecta a la víctima, ya que "[...] en estos casos el agresor no da la cara, no se identifica [...]. Estas agresiones desestabilizan a la víctima y acaban minando su autoestima. Además la víctima introyecta sentimientos de culpabilidad al no identificar claramente a su agresor o agresora" (p. 10). Asimismo, el robo, por pequeño que sea, debe ser totalmente rechazado porque es un hecho que, por lo demás, entra en el umbral de la ilegalidad y afecta la convivencia. Pero si existen esquemas de percepción que aprueban moral y culturalmente estos hechos se contribuye a la construcción de un imaginario que no considera esta forma de actuar incorrecta, por lo que Mockus (2002) explica, “[...] la convivencia consiste en buena parte 
en superar el divorcio entre ley, moral y cultura, es decir, superar la aprobación moral y/o cultural de acciones contrarias a la ley" (p. 21).

En cuanto a hostigar al estudiante mediante apodos, Collell y Escudé (2006) afirman que, "es preciso señalar la poca atención que se le suele prestar al maltrato verbal [...]. Contrariamente a lo que puede suponerse, las conductas de maltrato verbal y exclusión social tienen a largo plazo a un peor pronóstico para quien las sufre, que la agresión abierta" (p. 10).

Dentro de los hallazgos también se encuentra el acoso realizado por docentes, lo cual es más grave, porque como Chaux (2012) lo expone “[...] aumenta el riesgo de deserción escolar, sobre todo entre niñas que lo sufren. En segundo lugar, el maltrato por parte de docentes incentiva y legitima el maltrato por parte de compañeros" (p. 177). Referente a los acosadores solo algunos aceptan que ejercen ese rol, porque no reconocen que eso es intimidación, como lo expresan Prodócimo et al. (2014) "[...] El acto violento es considerado por muchos de los que lo cometen, como forma de obtener estatus o poder en el grupo" (p. 11). Dentro de las posibles razones de que no todos los que acosan se reconozcan como tales, Brank, Hoetger y Hazen (2012) explican que “[...] además de la baja empatía, no son capaces de entender que ese comportamiento vulnera a otros" (p. 217).

Para algunos de los hostigadores el imaginario de "poder" les da estatus de género si se es varón, fortaleciendo su masculinidad. Esto se debe al esquema de percepción que corresponde a la cultura patriarcal que aprueba, legitima y naturaliza algunos comportamientos del varón y que establece "[...] unas concepciones de lo femenino y lo masculino, la asignación de unos roles propios de cada uno de ellos, el otorgamiento de un status particular a cada una de esas posiciones de rol [...]" (Estrada, 2001, p. 25).

En lo concerniente a los observadores de estas situaciones, algunos de ellos no lo ven como un problema, esto lo revela Calvo y Ballester (2007) así: “[...] los observadores pueden llegar a aceptar la intimidación y considerarla como una experiencia normal de la vida escolar y, por lo tanto, algo que no se llega a cuestionar como ilegítimo" (p. 30), lo cual propicia el fortalecimiento del desequilibrio de poder y del imaginario de la naturalización del acoso escolar.

Con el propósito de afrontar la problemática, las IE han realizado talleres, convivencias, charlas y capacitaciones sobre la ley 1620 "por la cual se crea el sistema nacional de convivencia escolar y formación para el ejercicio de los derechos humanos [...]" (MEN, 2013 , p. 1). Aunque la existencia de la norma no garantiza comportamientos deseables, sí es una herramienta que sirve de parámetro para fortalecer los procesos que buscan transformaciones. Es así que: "cada vez que se legisla debería dispararse un proceso (preferiblemente voluntario) de cambio cultural y moral [...]" (Mockus, 2002, p. 33). Sin embargo, se pudo establecer que conflicto y acoso escolar son tenidos en cuenta por algunos miembros de la comunidad educativa como sinónimos, lo cual puede ocasionar que no se le dé un manejo apropiado a cualquiera de las situaciones.

Vale la pena subrayar que algunos de los padres y madres reconocen que a pesar de que las IE convocan a las actividades ellos no asisten, lo cual contribuye a que los procesos no se desarrollen cabalmente. El hecho de que los padres y madres no estén asumiendo la responsabilidad que les corresponde es un problema que cada día se ha ido agudizando y que aqueja no solo al departamento del Quindío, ya que muchos niños, niñas y adolescentes en Colombia durante su crianza no están contando con el amor, cuidado y acompañamiento de sus padres, y no es precisamente porque estos hayan fallecido como lo demuestra el 
estudio llevado a cabo en Colombia por Durán y Vayoles (2009), cuando expresan que "es preocupante que una tercera parte de los niños, niñas y adolescentes viva solo con uno de sus padres y que más de un millón no viva permanentemente con ninguno de los dos, así estén ellos vivos" (p. 781). Esto evidencia la vulneración de los derechos de los menores en cuanto a su desarrollo y protección. Aunque estas familias justifican la no presencia en la IE por motivos laborales, se puede ver que a muchos de ellos los orienta el imaginario social del consumismo, por lo tanto, satisfacer materialmente a sus hijos es suficiente.

Además, se pudo establecer que varios participantes se sienten mal cuando ven maltrato hacia algún miembro de la IE, lo que les genera sentimientos de rabia, tristeza, indignación, impotencia, dolor, temor, angustia y depresión entre otros. Cabe destacar la idea suicida que manifestó un participante. Al respecto Collell y Escudé (2006) hacen referencia acerca de lo que puede generar la intimidación a las personas que la sufren, dentro de los efectos se encuentran "[...] sentimientos de infelicidad, bajos niveles de confianza y autoestima, desajuste escolar, bajo rendimiento académico etc. [...] estados psicológicos más estresantes como pueden ser altos niveles de ansiedad, depresión o ideación suicida" (p. 12). Estos estados, aunque no brindan un conocimiento amplio, permiten interpretar en parte las habilidades emocionales de las cuales Rodicio e Iglesias (2011) distinguen dos grandes componentes: “a) la capacidad de autorreflexión [...]; b) la habilidad de reconocer lo que los demás están pensando y sintiendo [...]” (p. 114).

También se identificaron algunos imaginarios por los que se promueve o justifica el hostigamiento, entre ellos tener mala reputación, no cumplir con los cánones de belleza por presentar capacidades diversas, por expresar la homosexualidad de manera pública, etcétera. Estas situaciones o condiciones que hacen parte de la diversidad humana, en muchas ocasiones son asumidas por la sociedad como sinónimos de inferioridad. Lo cual conlleva a que el maltrato hacia estas personas sea aceptado como algo natural. Algunos teóricos tratan de dar una explicación a la problemática, entre ellos Pintos (1995) quien afirma que el "restablecimiento de un darwinismo social (absoluta desprotección de los más débiles de todo tipo), con una clara tendencia a presentar los procesos sociales como sometidos a leyes naturales" (p. 104). Por tal motivo, es relevante desnaturalizar la intimidación que está enraizada en la cultura y habita en el inconsciente de las personas.

Desde esta perspectiva, conseguir verdaderamente un cambio y resolver el problema solo se logra en gran parte " [...] cuando por lo menos un sector amplio de la población toma conciencia del mecanismo de poder que legitima la práctica social cuestionada y se generan nuevas prácticas sociales tendientes a transformar, así sea lentamente, la situación cuestionada [...]" (Vasco et al., 1999, p. 108). Aunque es claro que esto no se consigue de manera sencilla, porque "no hay fenómeno de dominación más sutil y más potente que el que se consigue hacerse totalmente invisible y totalmente impalpable [...]" (Ibáñez, 2005, p. 145), como es el caso de los imaginarios que hacen presencia en la intimidación escolar.

Para lograr el propósito deseado se recurrió al pensamiento crítico como estrategia pedagógica, el cual en esta investigación jugó un papel importante en el proceso de intervención. No obstante hay que reconocer que para alcanzar el nivel del pensamiento crítico también se requiere un proceso de formación que estimule dicho desarrollo, pero infortunadamente la educación en Colombia muy poco apunta a ello, y en este estudio se pudo constatar una vez más, pues la mayoría de las y los participantes mostraron que alcanzaban solamente el pensamiento reflexivo, que Villarini (s.f.) define en las siguientes palabras: " [...] consiste en el empleo deliberado y sistemático de nuestros recursos mentales 
a la luz del propósito o meta de entender, explicar, manejar, decidir o crear algo. [...] es el pensamiento instrumental por excelencia" (p. 38).

Esto se pudo evidenciar porque en pocas actividades algunos participantes alcanzaron las dimensiones (lógica, sustantiva, contextual, dialógica y pragmática) que conforman el pensamiento crítico, las cuales permiten "criticar nuestro propio proceso de construcción de conocimiento, es decir a asumir una actitud crítica que nos eleva de la mera conciencia a autoconciencia y nos permite constantemente cuestionar y rehacer nuestra actividad" (Villarini, 2007, p. 251), para lo cual se debe realizar el cuestionamiento a la información que se recibe cotidianamente, a las creencias de todo tipo, a las ideologías propias y de los otros y a los mecanismos de poder que hacen presencia en todos los espacios de la sociedad (Ruiz y Chaux, s.f.).

Aunque es importante el pensamiento reflexivo para llegar al pensamiento crítico, no es suficiente para que una sociedad logre transformar los imaginarios que la afectan negativamente, como tampoco es suficiente para desarrollar a cabalidad las competencias ciudadanas que requiere una nación en la formación de verdaderos ciudadanos democráticos.

Sin embargo esta situación era predecible, y por ello no fue un obstáculo para alcanzar, aunque no en el nivel deseado, el objetivo general de la investigación y los objetivos específicos, pudiéndose implementar lo planeado y lograr que el grupo de "observadores" de situaciones de acoso comprendieran la importancia de los imaginarios sociales y del pensamiento crítico para hacer visible lo que no es tan evidente, tanto en el "yo" como en el "otro", y así poder transformar con acciones noviolentas los imaginarios sociales que posibilitan la existencia del hostigamiento escolar. Es así que después del proceso de intervención varios participantes coincidieron en que era destacable el compromiso del estudiantado que conformaba parte del grupo que lideró la intervención —además, había mejorado la convivencia entre el estudiantado, sin desconocer que es necesario mejorar aún más-, pero, infortunadamente, no se puede decir lo mismo en cuanto a la relación con algunos profesores que sigue enmarcada en el irrespeto y la intolerancia.

Para alcanzar los cambios indicados, y con ello la iniciación de un buen proceso, se contó con el compromiso de algunas profesoras con quienes el grupo de estudiantes planearon e implementaron en sus aulas diferentes acciones noviolentas, y en las que siempre tenían que recordar que son "[...] formas que rechazan la pasividad — dirigida a aguantar-y reivindica un camino creativo para construir mejores realidades" (Fernández, 2003, p. 42).

Considerando que la intimidación escolar es una dinámica grupal, las estrategias para enfrentarla también fueron diseñadas desde ese enfoque, contribuyendo así a que los participantes del grupo para la intervención entretejieran afectos y construyeran confianzas, elementos fundamentales para el fortalecimiento de cualquier proceso social y en los cuales los imaginarios juegan un papel importante, según lo explica Pintos (2005), así "los diferentes imaginarios sociales con los que se construye una relación de 'confianza' [...]. No se define por lo que uno hace (fiarse, confiar), sino por cómo los demás lo consideran (digno de confianza, fiable)" (p. 49).

Es así que las y los estudiantes que lideraron este proceso, de acuerdo con lo que previamente habían planeado cuando se presentara algún episodio de intimidación, intervenían en grupo para impedir el hostigamiento. Si era el caso, pedían al profesor interrumpir la clase hasta que cesara el acoso. Además, estos mismos estudiantes, con el acompañamiento de algunas profesoras, organizaron mesas redondas y conversatorios 
en los que participaban todos los integrantes del aula para hablar sobre las situaciones de acoso vividas allí y explicar o aclarar los conceptos de acoso escolar, imaginarios sociales, pensamiento crítico y noviolencia, tomando como ejemplo, en lo posible, casos reales en cuestión.

Otro aspecto a destacar en el proceso de intervención fue lograr que en algunos estudiantes que eran parte del grupo para la intervención, se estimularan las neuronas espejo y que en una situación de acoso se unieran al grupo que estaba evitando el episodio. Sobre estas neuronas, García (2008) precisa que: “[...] son un tipo particular de neuronas que se activan cuando un individuo realiza una acción, pero también cuando él observa una acción similar realizada por otro individuo. [...] Le permite ponerse en lugar de otros, leer sus pensamientos, sentimientos y deseos, lo que resulta fundamental en la interacción social" (p. 7).

Según García (2008) las neuronas espejo no solo se activan cuando se observan hechos de manera presencial, sino también cuando se observan a través de videos. Lo anterior demuestra que si en las IE se trabaja transversalmente procesos para mejorar la convivencia, debe hacerse de manera planeada, coordinada, con objetivos claros y no cortoplacistas; de esta manera se pueden lograr transformaciones culturales. Estos procesos no pueden ser desarticulados, pues para que se produzcan las transformaciones deseadas se debe "[...] generar una cultura que incorporada a la cotidianidad de la institución educativa deconstruya inquebrantablemente y comprometidamente las viejas maneras de ser, estar, sentir, explicar y hacer" (Ghiso y Ospina, 2010, p. 553).

En lo concerniente a factores limitantes en este proceso investigativo, se puede decir que el tiempo fue uno de ellos, dado que este estudio debía regirse por calendarios académicos establecidos por las IE, lo que generó restricciones para obtener mayores posibilidades de intervención, retroalimentación y evidencias de los resultados.

Según lo hallado en este estudio, y para futuras investigaciones, se sugiere que en procesos en los cuales haya intervención se incluya al profesorado y adultos responsables del cuidado y crianza de los niños, niñas y adolescentes, porque muchos de los esquemas de percepción que sustentan la actuación de los miembros de las comunidades educativas hacen parte de los imaginarios atávicos, siendo estos "[...] aprendizajes colectivos [...] que se transmiten o heredan de forma inconsciente, se mantienen de forma recurrente y, respecto de los que definen la cultura hegemónica de hoy, tienen su fuente en la cultura patriarcal" (Martínez, 2012, p. 31). Por lo cual, los adultos que hacen parte del contexto al que pertenece el estudiantado juegan un papel fundamental para transformar los imaginarios que actualmente están orientando los comportamientos violentos de algunos/as estudiantes y profesores.

\section{CONCLUSIÓN}

Dentro de los aportes que deja este estudio, y aclarando que no hay intención de minimizar la problemática del acoso escolar, las IE pueden aprovechar la intervención que deben realizar como una oportunidad para fortalecer el desarrollo de las competencias ciudadanas, puesto que no es conveniente para la sociedad que estos procesos sean trabajados desde y para la escuela. Es importante traspasar estos límites de tiempo y de espacio para que esos ciudadanos que se encuentran en proceso de formación en las 
escuelas entiendan, con su pensar crítico, que los imaginarios sociales son creados por la mente humana y habitan en ella, y que pueden ser fortalecidos o trasformados según se requiera. De esta forma, también se contribuye a que el estudiantado comprenda la necesidad de actuar de manera autónoma, ética, solidaria y propositiva tanto en los ámbitos social, político y económico como en la esfera pública y la privada, para que así, en unión con otros, construyan una sociedad pacífica y democrática en la cual todos y cada uno de sus miembros son respetados y aceptados en su diversidad.

\section{REFERENCIAS BIBLIOGRÁFICAS}

Baeza, M. A. (2011). Memoria e imaginarios sociales. Imagonautas: revista interdisciplinaria sobre imaginarios sociales, 1(1), 76-95.

Brank, E., Hoetger, L., \& Hazen, K., (2012). Bullying. The Annual Review of Law and Social Science, 8, 213-230. doi:10.1146/annurev-lawsocsci-102811-173820.

Calvo, A., \& Ballester, F. (2007). Acoso escolar: Procedimientos e intervención. Madrid: Editorial EOS.

Chaux, E. (2007). Aulas en Paz: Resultados Preliminares de un Programa Multi-Componente. Recuperado de http://www.mineducacion.gov.co/cvn/1665/articles-164318_pdf_1.pdf

Chaux, E. (2012). Educación, convivencia y agresión escolar. Bogotá: Ediciones uniandes.

Collell, J., \& Escudé, C. (2006). El acoso escolar: un enfoque psicopatológico. Anuario de psicología clínica y de la salud, 2, 9-14. Recuperado de http://institucional.us.es/apcs/doc/APCS_2_esp_914.pdf

Colombia aprende. (2011). Orientaciones para la institucionalización de las competencias ciudadanas. Cartilla1. Recuperado de http://www.colombiaaprende.edu. co/html/ micrositios/1752/articles-314549_recurso_2.pdf

Cowie, H., \& Fernández, J. (2006). Ayuda entre iguales en las escuelas: desarrollo y retos. Revista electrónica de investigación psicoeducativa 4(2), 291-310.

Domínguez, J., López, A., Pino, M., \& Álvarez, E. (2013). Violencia escolar: la díada interpersonal profesorado-alumnado. European Journal of investigation in Healt, Psichology and Education, $3(2), 75-86$.

Durán,E.,\&Vayoles,E. (2009).Perfildelosniños, niñas y adolescentes sincuidadoparental enColombia. Revista Latinoamericana de ciencias sociales niñez y juventud, 7(2), 761-783. Recuperado de http://www.scielo.org.co/scielo.php?pid=S1692-715X2009000200008\&script=sci_arttext

Estrada, A. (2001). El patriarcado, fuente simbólica reguladora de la estructura simbólica de la cultura. Bogotá: Corporación Viva la Ciudadanía.

Feldman, M., Donato, I., \& Wright, J. (2013). Bullying and Suicide: A Public Health Approach. Journal of Adolescent Health, 53(1), 51-53.

Fernández, C. (Ed.). (2003). Desvelando el poder de la influencia sutil... Hacia una pedagogía de la noviolencia. En Aquí estoy país (Ed.), El poder de la fragilidad. (pp. 21-29). Bogotá: Kimpres Ltda.

Forero-Londoño, O. (2011). La violencia escolar como régimen de visibilidad. Magis, Revista Internacional de Investigación en Educación, 4(8), 399-413.

García, A. (2009). El acoso entre iguales. Innovación y experiencias educativas, 14. Recuperado de http://www.csi- csif.es/andalucia/modules/mod_ense/revista/pdf/ Numero_14/ ALICIA\%ELENA_GARCIA_2.pdf

García, E. (2008). Neuropsicología y educación. De las neuronas espejo a la teoría de la mente. Revista de psicología y educación, 1(3), 69-90.

García, J., González, M., \& Ballesteros V. (2001). Introducción a la investigación en educación 
(Tomo I). Madrid: UNED.

Gento, S. (2006). Propuesta para una acción educativa de calidad en el tratamiento de la diversidad. Revista complutense de educación, 17(2), 13-34.

Ghiso, A., \& Ospina, V. (2010). Naturalización de la intimidación entre escolares: un modo de construir lo social. Revista latinoamericana de ciencias sociales niñez y juventud, 8(1), 535-556.

Hernández, R., Fernández, C., \& Baptista, P., (2010). Metodología de la investigación. México: Mac Graw Hill

Ibáñez, T. (2005). Contra la dominación. Barcelona: Gedisa.

Instituto Colombiano para el Fomento de la Educación Superior. (2013). Resultados nacionales censales de competencias ciudadanas SABER $3^{\circ}, 5^{\circ}$ y $9^{\circ}$, 2012. Recuperado de http://www2. icfesinteractivo.gov.co/SaberCensal359Reportes_2013-web/seleccionReporteIdDane.jspx;jsess ionid=1376d3b9df19dfb73e8d9640aff5

Loredo, A., Perea, A., \& López, G. (2008). "Bullying": acoso escolar. La violencia entre iguales. Acta pediátrica de México, 29(4). Recuperado el 15 de diciembre de 2014 desde http://www. medigraphic.com/pdfs/actpedmex/apm-2008/apm084e.pdf

Martínez, C. (2012). De nuevo la vida. El poder de la noviolencia y las transformaciones culturales. Bogotá: Uniminuto.

Ministerio de Educación Nacional. (2002). Decreto 3020. Criterios y procedimientos para organizar las plantas de personal docente y administrativo. Recuperado de http://www.mineducacion.gov. co/1621/article-104848.html

Ministerio de Educación Nacional. (2013). Ley 1620. Creación del sistema nacional de convivencia escolar. Recuperado de http://www.mineducacion.gov.co/1621/propertyvalue-31214.html

Mockus, A. (2002). Convivencia como armonización de ley, moral y cultura. Perspectivas XXXI(1), 19-37.

Mockus, A., \& Cancino, D. (2012). Prólogo. En E. Chaux, Educación, convivencia y agresión escolar. Bogotá: Ediciones Uniandes.

Olweus, D. (1993). Conductas de acoso y amenaza entre escolares. Madrid: Morata.

Organización de Estados Iberoamericanos para la Educación, la Ciencia y la Cultura. (2007). Violencia escolar: el maltrato entre iguales en la secundaria obligatoria, 199. Recuperado de http://www.oei.es/oeivirt/Informeviolencia.pdf

Organización Mundial de la Salud. (2002). Informe mundial sobre la violencia y la salud. Washington, D.C.: Biblioteca OPS. Recuperado de http://www.who.int/violence_injury_prevention/violence/ world_report/es/summary_es.pdf

Pintos, J. (1995). Orden social e imaginarios sociales (Una propuesta de investigación). Papers. Revista de sociología. 1995, 101-127. Recuperado de http://www.raco.cat/index.php/papers/ article/viewFile/25267/58550

Pintos, J. (2004). Inclusión-Exclusión. Los imaginarios sociales de un proceso de construcción social. SÉMATA, Ciencias Sociais e Humanidades, 16, 17-52.

Pintos, J. (2005). Comunicación, construcción de la realidad e imaginarios sociales. Revista internacional de filosofía latinoamericana y teoría social. Utopía y praxis latinoamericana, año 10(29), 37-65.

Pintos, J. (2014). Algunas precisiones sobre el concepto de imaginarios sociales. Revista Latina de sociología 4, 1-11.

Prodócimo, E., Gonçalves, R., Rodrigues, R., \& Bognoli, P. (2014). Violencia escolar: reflexiones sobre los espacios de ocurrencia. Revista Electrónica de Investigación Educativa, 16(2), 1-15.

Rodicio, M., \& Iglesias, M. (2011). El acoso escolar. Diagnóstico y prevención. Madrid: Biblioteca Nueva.

Román, M., \& Murillo, J. (2011). América latina: Violencia entre estudiantes. Revista Cepal, 104. Recuperado de: http://www.cepal.org/publicaciones/xml/3/44073/RVE104RomanMurillo.pdf

Ruiz, A., \& Chaux, E. (s.f). La formación de competencias ciudadanas. Recuperado de http:// 
publicacionesfaciso.uniandes.edu.co/sip/data/pdf/formacion_de_competencias_ciudadanas.pdf

Valadez, I. (2008). Violencia escolar: Maltrato entre iguales en escuela de secundaria de la zona metropolitana de Guadalajara. Recuperado de cvsp.cucs.udg.mx/drupal6/documentos/ violencia_escolar_libro.pdf

Valadez, I., Amezcua, R., González, N., Montes, R., \& Vargas, V. (2011). Maltrato entre iguales e intento suicida en sujetos adolescentes escolarizados. Revista Latinoamericana de Ciencias Sociales, Niñez y Juventud, 2(9), 783-796.

Vargas, Z. (2009). Investigación aplicada: una forma de conocer las realidades con evidencia científica. Educación, 33(1), 155-165.

Vasco, C., Bermúdez, A., Escobedo, H., Negret, J., \& León, T. (1999). El saber tiene sentido. Una propuesta de integración curricular. Bogotá: Antropos.

Villarini, A. (2007). Teorías que necesitamos los investigadores y practicantes de la educación: una reflexión crítica. Contribución al debate. Cultura y Educación, 9(3), 249-255.

Villarini, A. (s.f). Teoría y pedagogía del pensamiento crítico. Perspectivas psicológicas, 3-4 (año IV), 35-42. 